\title{
Anatomia do Lenho de Cinco Espécies Comercializadas como 'sucupira'
}

\author{
Warley Felício Soares ${ }^{1}$, Luiz Eduardo de Lima Melo ${ }^{2}$, Pedro Luiz Braga Lisboa ${ }^{3}$ \\ ${ }^{1}$ Departamento de Tecnologia da Madeira, Universidade do Estado do Pará - UEPA, Belém/PA, Brasil \\ ${ }^{2}$ Departamento de Ciências Florestais, Universidade Federal de Lavras - UFLA, Lavras/MG, Brasil \\ ${ }^{3}$ Coordenação de Botânica - CBO, Museu Paraense Emilio Goeldi - MPEG, Belém/PA, Brasil
}

\begin{abstract}
RESUMO
A análise comparativa da anatomia do lenho das espécies Bowdichia nitida Spruce ex Benth, Diplotropis brasiliensis (Tul.) Benth., Diplotropis martiusii Benth., Diplotropis purpurea (Rich.) Amshoff e Diplotropis racemosa (Hoehne) Amshoff é descrita no presente trabalho, visando a determinar as principais características anatômicas necessárias à distinção dessas espécies. Qualitativamente, as características anatômicas mais importantes para a diferenciação do lenho dessas espécies foram: camadas de crescimento, estratificação dos raios, arranjo dos vasos e fileira marginal dos raios. Quantitativamente, a análise estatística mostrou que os parâmetros anatômicos mais importantes no agrupamento das espécies foram: diâmetro dos elementos de vasos, largura dos raios em micrometros, comprimento, espessura da parede e largura das fibras. Verificou-se que a anatomia do lenho pode ser utilizada como importante subsídio na identificação das espécies estudadas.
\end{abstract}

Palavras-chave: anatomia do lenho, sucupira, comércio de madeira.

\section{Anatomy of Five Wood Species Marketed as 'Sucupira'}

\begin{abstract}
In this study, we describe the comparative anatomical analysis of the following wood species: Bowdichia nitida Spruce ex Benth, Diplotropis brasiliensis (Tul.) Benth., Diplotropis martiusii Benth., Diplotropis purpurea (Rich.) Amshoff, and Diplotropis racemosa (Hoehne) Amshoff. The aim was to determine the main anatomical characteristics to distinguish between these wood species. Qualitative analysis showed that the most important anatomical characteristics for the differentiation of these wood species were growth rings, stratification rays, arrangement of vases, and row marginal rays. Quantitative statistical analysis showed that the major anatomical parameters in the clustering of species were diameter of vessel elements, length of rays in micrometers, length, wall thickness, and width of fibers. We verified that wood anatomy can be of great importance in identifying the species studied.
\end{abstract}

Keywords: wood anatomy, 'Sucupira', timber trade. 


\section{INTRODUÇÃO}

A exploração e o processamento industrial de madeira estão entre as principais atividades econômicas da Amazônia, ao lado da mineração e da agropecuária. Esse fato está ligado principalmente à oferta abundante de madeira de diversas espécies.

Notadamente, no que diz respeito à utilização de espécies madeireiras nativas da Floresta Amazônica, pode-se dizer que o verdadeiro potencial desse material biológico não tem sido devidamente explorado. Um dos fatores culminantes para esse fato deve-se à inadequação do uso da madeira, principalmente relacionado a erros de identificação das espécies comercializadas, o que compromete o uso final dessa matéria-prima. Gomes et al. (2002) citam, ainda, a introdução de espécies madeireiras no mercado de aspectos semelhantes às tradicionalmente utilizadas, embora com qualidade inferior para a aplicação a que se destinam.

Segundo Martins-da-Silva (2002), uma espécie chega a ter até dez nomes vernaculares e existe ainda a relação de diversos nomes vulgares para um mesmo táxon. Kanashiro (2002) afirma que, por serem semelhantes, as espécies são confundidas e exploradas de forma desordenada e não sustentável; o autor afirma ainda que a distinção das espécies de forma clara e didática é necessária para minimizar prejuízos econômicos e colaborar para o controle da manutenção da biodiversidade.

Nesse contexto, o estudo da anatomia da madeira nos permite conhecer os possíveis empregos da madeira, a partir de indicadores técnicos e do conhecimento de suas reais possibilidades de uso, proporcionando agregação de valor aos seus produtos, além de diminuir os equívocos cometidos na comercialização destas madeiras (Burger \& Richter, 1991). Segundo Mady (2007), o estudo da anatomia do lenho tem grande importância nas regiões tropicais, pois ainda existe um elevado número de espécies que tem suas características pouco conhecidas.

As madeiras comumente conhecidas por 'sucupira’ pertencem à família Fabaceae subfamília Faboideae e são representadas por espécies pertencentes aos gêneros Bowdichia Kunth, constituído por cerca de 20 espécies, e Diplotropis
Benth., com número aproximado de 22 espécies, sendo $B$. nitida, B. virgilioides, D. purpurea, D. racemosa e $D$. martiusii as espécies que comumente recebem essa designação popular (Umaña \& Alencar, 1993; Heywood, 1996). São espécies que apresentam madeira de alta densidade e lenho resistente, sendo por isso utilizadas para fabricação de dormentes, construção civil e naval, tacos e tábuas para assoalhos, móveis, faqueados decorativos, etc. (Silva et al., 1977; Loureiro et al., 2000). Segundo Mainieri \& Chimelo (1989) e Jankowsky et al. (1990), as diferenças na estrutura anatômica existentes entre estas espécies são pouco perceptíveis, sendo por isso comercializadas como variações da própria madeira.

A partir do exposto, objetivou-se descrever a anatomia da madeira de cinco espécies comercializadas como 'sucupira', disponíveis no acervo da Xiloteca Walter Alberto Egler, do Museu Paraense Emílio Goeldi, e verificar se os caracteres anatômicos do lenho podem ser utilizados para segregar essas espécies.

\section{MATERIAL E MÉTODOS}

As amostras utilizadas neste trabalho foram coletadas do acervo da xiloteca Walter Alberto Egler, do Museu Paraense Emílio Goeldi, com respectivo material botânico correspondente no Herbário João Murça Pires da mesma instituição (Tabela 1). Os corpos de prova foram obtidos com tamanho aproximado de $2 \times 2 \times 2 \mathrm{~cm}$, respeitando os planos transversal, longitudinal tangencial e longitudinal radial. Todo o trabalho foi desenvolvido no laboratório de Anatomia da Madeira do Museu Paraense Emílio Goeldi.

A descrição macroscópica do lenho foi realizada com uma lupa de $10 \times$ de aumento, seguindo as normas do Instituto Brasileiro do Meio Ambiente e dos Recursos Naturais Renováveis - IBAMA (1992). A descrição microscópica seguiu as normas do International Association of Wood Anatomists Committee - IAWA COMMITTEE (1989).

Os corpos de prova obtidos foram cozidos em água e glicerina na proporção de 4:1 até atingirem a condição ideal para o seccionamento. As secções histológicas foram obtidas em micrótomo de deslize (Leitz 1208), com espessura entre 15 e $18 \mu \mathrm{m}$ nos 
Tabela 1. Espécies analisadas com seus respectivos números de registros

Table 1. Species analyzed with their respective registration numbers.

\begin{tabular}{lcc}
\multicolumn{1}{c}{ Nome Científico } & MGX & MGH \\
\hline Bowdichia nitida Spruce ex Benth. & 5391 & 134191 \\
Diplotropis brasiliensis (Tul.) Benth. & 4402 & 107851 \\
Diplotropis martiusii Benth. & 4554 & 114321 \\
Diplotropis purpurea (Rich.) Amshoff & 5267 & 134520 \\
Diplotropis racemosa (Hoehne) Amshoff & 5218 & 134505 \\
\hline
\end{tabular}

MGX - número de registro da amostra na xiloteca; MGH - número de registro das amostras no herbário.

planos transversal, longitudinal tangencial e radial. Os cortes foram então clarificados em hipoclorito de sódio $60 \%$ e corados com safranina hidroalcoólica $1 \%$ (Johansen, 1940). Após a coloração e a desidratação dos cortes, foram montadas lâminas provisórias para mensuração dos elementos anatômicos, bem como lâminas permanentes em bálsamo-do-canadá (diluído em acetato na proporção de 1:3) para documentação fotomicrográfica das estruturas anatômicas. Para a mensuração dos elementos anatômicos dissociados, foi preparado material macerado segundo o método de Franklin (1945).

Realizaram-se, para cada variável analisada (elementos de vasos - frequência/ $/ \mathrm{mm}^{2}$, comprimento e diâmetro; raios - frequência $/ \mathrm{mm}$ linear, altura em número de células e em micrômetros, largura em número de células e em micrômetros; fibras - comprimento, espessura da parede e largura do lume), 30 contagens e medições. As contagens e mensurações foram efetuadas sob microscópio, acoplado à câmera de vídeo com auxílio do programa Visilog 5.1, nos cortes histológicos e no material macerado.

A documentação fotográfica macroscópica foi realizada na superfície transversal do corpo de prova, por meio de um estereomicroscópio ligado ao computador com o programa Motic plus 2.0. As imagens microscópicas foram realizadas usando um microscópio ligado ao computador com o programa Aver tv.

O teste de Shapiro-Wilk W foi utilizado para testar a normalidade das amostras (Zar, 1996). Realizou-se análise de variância e, quando diferença significante foi detectada, aplicou-se o teste de ScottKnott a 5\% de probabilidade, para comparação entre as médias das características anatômicas quantitativas das cinco espécies estudadas. Para verificar a formação de grupos distintos, foi realizada uma análise de agrupamento, baseado em uma matriz de distância, usando os caracteres anatômicos quantitativos das espécies. A análise dos componentes principais foi utilizada para ordenar as espécies e os caracteres anatômicos quantitativos, além de evidenciar os fatores de maior variância (Ludwig \& Reynolds, 1988). Na análise de agrupamento e na de componentes principais, foram utilizados os parâmetros anatômicos: frequência dos vasos por $\mathrm{mm}^{2}$; comprimento dos elementos de vaso $(\mu \mathrm{m})$; diâmetro dos elementos de vaso $(\mu \mathrm{m})$; frequência dos raios (número $\mathrm{mm}$ linear); altura dos raios $(\mu \mathrm{m})$; altura dos raios (número de células); largura dos raios $(\mu \mathrm{m})$; largura dos raios (número de células); comprimento das fibras $(\mu \mathrm{m})$; diâmetro do lume das fibras $(\mu \mathrm{m})$; espessura da parede das fibras $(\mu \mathrm{m})$; largura da fibra $(\mu \mathrm{m})$, e fração parede da fibra (\%).

\section{RESULTADOS E DISCUSSÃO}

\subsection{Descrição anatômica das espécies}

\section{Bowdichia nitida Spruce ex Benth.}

- Análise macroscópica

Parênquima axial: visível a olho nu; predominantemente paratraqueal aliforme losangular, formando curtas confluências, ocorre também em finas linhas marginais e unilaterais (Figura 1a). Raios: visíveis a olho nu, estratificação regular. Poros: visíveis a olho nu, porosidade difusa, arranjo com padrão indefinido, agrupados em múltiplos de 2 a mais; apresenta alguns poros obstruídos por substância de cor escura e, em menor quantidade, por substância esbranquiçada (Figura 1a). Camadas de crescimento: individualizadas por parênquima marginal (Figura 1a). 


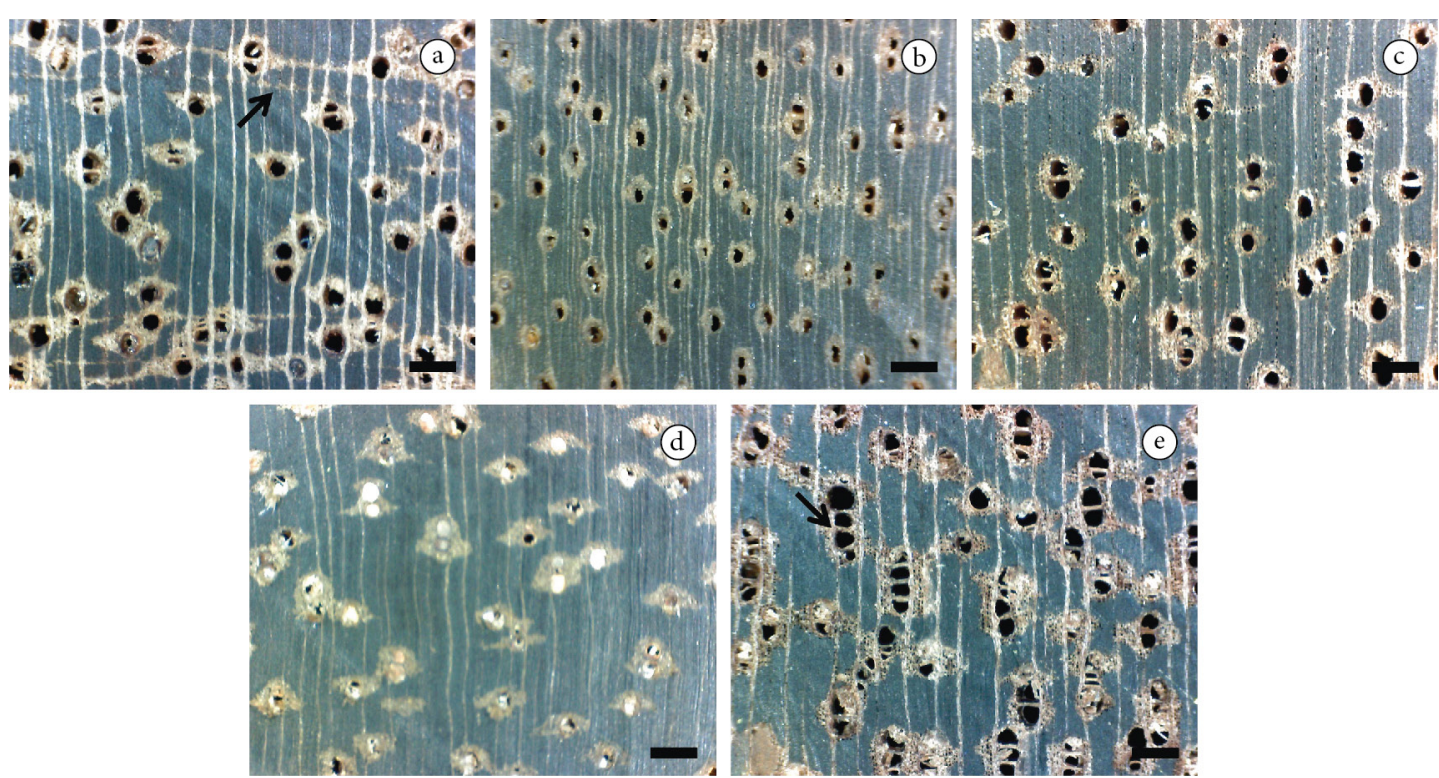

Figura 1. Vista macroscópica da secção transversal. a) Bowdichia nitida Spruce ex Benth, evidenciando camadas de crescimento (seta). b) Diplotropis brasiliensis (Tul.) Benth. c) Diplotropis martiusii Benth. d) Diplotropis purpurea (Rich.) Amshoff. e) Diplotropis racemosa (Hoehne) Amshoff, evidenciando arranjo radial dos vasos (seta). Barra $=500 \mu \mathrm{m}$.

Figure 1. Transection macroscopic view. a) Bowdichia nitida Spruce ex Benth, showing the growth rings (arrows). b) Diplotropis brasiliensis (Tul.) Benth. c) Diplotropis martiusii Benth. d) Diplotropis purpurea (Rich.) Amshoff. e) Diplotropis racemosa (Hoehne) Amshoff, showing the radial arrangement of the vessels (arrows). Scale bars $=500 \mu \mathrm{m}$.

\section{- Análise microscópica}

Vasos: porosidade difusa; arranjo com padrão indefinido; solitários na maioria, ocorrem também múltiplos de 2 a mais; forma da secção arredondada (Figura 2a); placa de perfuração simples; pontoações intervasculares alternas; pontoações radiovasculares semelhantes às intervasculares; largura variando entre 110 e $370 \mu \mathrm{m}$ (média de 247,33 $\mu \mathrm{m}$ ); comprimento variando entre 280 e $510 \mu \mathrm{m}$ (média de $386 \mu \mathrm{m})$; frequência por $\mathrm{mm}^{2}$ variando de 2 a 8 (média de 3,83). Fibras: libriformes; comprimento variando de 1.020 a $2.360 \mu \mathrm{m}$ (média de 1713,67 $\mu \mathrm{m}$ ); largura do lume variando de 0,84 a 4,76 $\mu \mathrm{m}$ (média de 1,64 $\mu \mathrm{m}$ ); espessura da parede variando de 3,92 a $11,86 \mu \mathrm{m}$ (média de $7,56 \mu \mathrm{m}$ ). Parênquima axial: paratraqueal aliforme de aleta grossa formando curtas confluências, ocorre também marginal e unilateralmente; três a quatro células por strand de parênquima. Raios: corpo das células procumbentes com uma fila de células marginais quadradas (Figura 4a); todos os raios estratificados (Figura 3a); altura em micrômetros variando de 220 a $580 \mu \mathrm{m}$ (média de 323,33 $\mu \mathrm{m}$ ); altura em número de células variando de 8 a 21 (média de 13,60); largura em micrômetros variando de 20 a $80 \mu \mathrm{m}$ (média de 46,33 $\mu \mathrm{m})$; largura em número de células variando de 1 a 4 (média de 3,20 ); frequência por $\mathrm{mm}$ variando de 6 a 10 (média de 7,77) (Figura 3a). Camadas de crescimento: individualizadas por parênquima marginal.

\section{Diplotropis brasiliensis (Tul.) Benth.}

- Análise macroscópica

Parênquima axial: visível a olho nu; paratraqueal aliforme e unilateral (Figura 1b). Raios: visíveis a olho nu, não estratificados. Poros: visíveis a olho nu, porosidade difusa, arranjo com padrão indefinido, agrupados em múltiplos de 2 a mais, alguns totalmente obstruídos por substancia de cor esbranquiçada (Figura 1b). Camadas de crescimento: indistintas.

- Análise microscópica

Vasos: porosidade difusa; arranjo com padrão indefinido; solitários na maioria, ocorrem também múltiplos de 2 a mais; forma da secção arredondada (Figura 2b); placa de perfuração simples; pontoações 

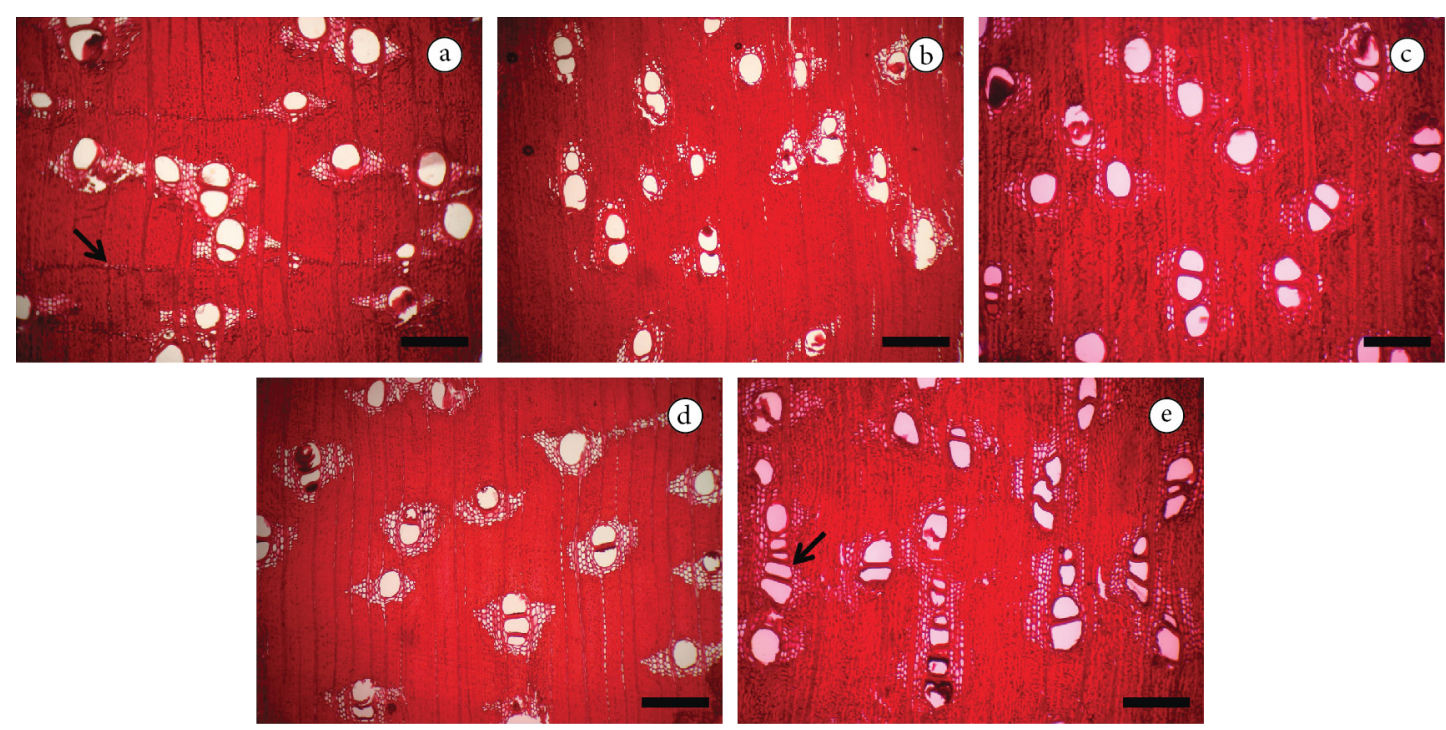

Figura 2. Vista microscópica da secção transversal. a) Bowdichia nitida Spruce ex Benth, evidenciando camadas de crescimento (seta). b) Diplotropis brasiliensis (Tul.) Benth. c) Diplotropis martiusii Benth. d) Diplotropis purpurea (Rich.) Amshoff. e) Diplotropis racemosa (Hoehne) Amshoff, evidenciando arranjo radial dos vasos (seta). Barra $=500 \mu \mathrm{m}$.

Figure 2. Transection microscopic view. a) Bowdichia nitida Spruce ex Benth, showing the growth rings (arrows). b) Diplotropis brasiliensis (Tul.) Benth. c) Diplotropis martiusii Benth. d) Diplotropis purpurea (Rich.) Amshoff. e) Diplotropis racemosa (Hoehne) Amshoff, showing the radial arrangement of the vessels (arrows). Scale bars $=500 \mu \mathrm{m}$.
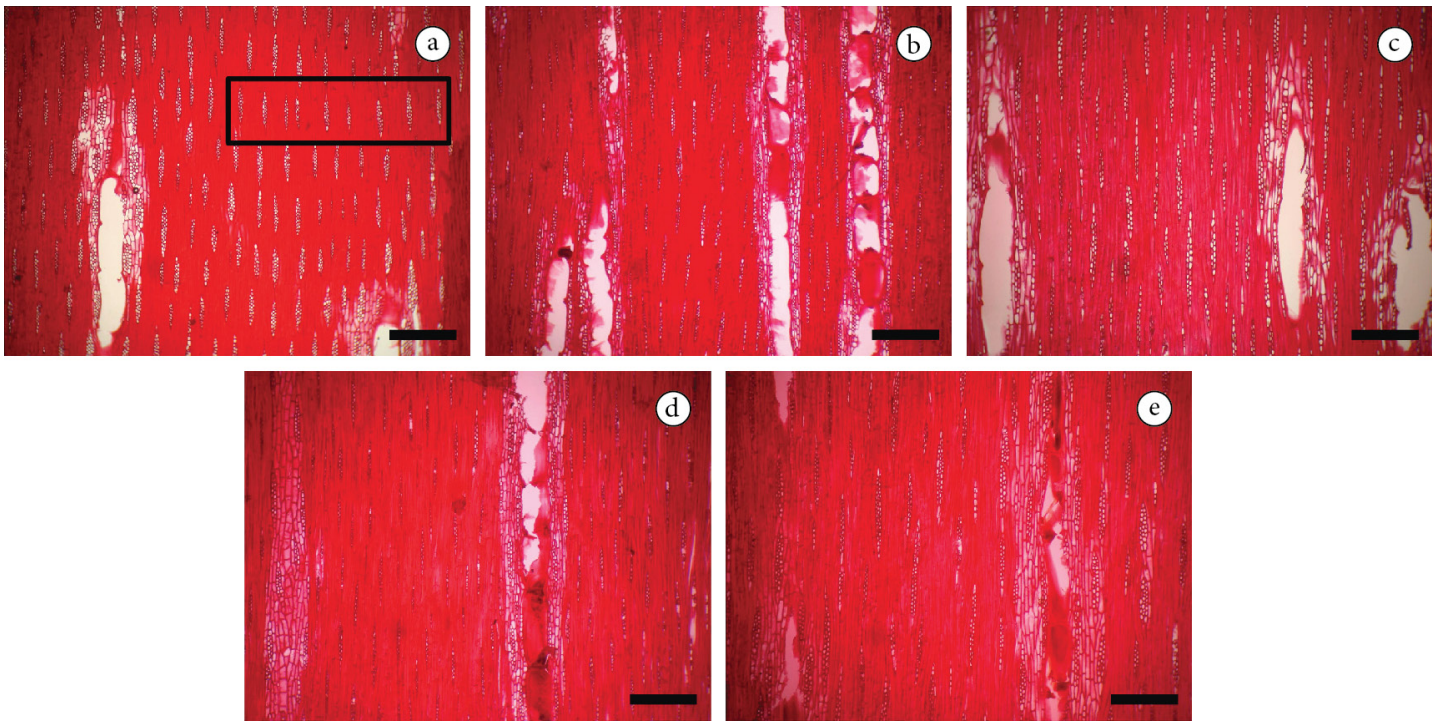

Figura 3. Vista microscópica da secção longitudinal tangencial. a) Bowdichia nitida Spruce ex Benth, evidenciando estratificação dos raios (retângulo). b) Diplotropis brasiliensis (Tul.) Benth. c) Diplotropis martiusii Benth. d) Diplotropis purpurea (Rich.) Amshoff. e) Diplotropis racemosa (Hoehne) Amshoff. Barra $=500 \mu \mathrm{m}$.

Figure 3. Longitudinal tangential microscopic view. a) Bowdichia nitida Spruce ex Benth, showing the stratification of the ray (rectangle). b) Diplotropis brasiliensis (Tul.) Benth. c) Diplotropis martiusii Benth. d) Diplotropis purpurea (Rich.) Amshoff. e) Diplotropis racemosa (Hoehne) Amshoff. Scale bars $=500 \mu \mathrm{m}$.

intervasculares alternas; pontoações radiovasculares semelhantes às intervasculares; largura variando de 110 a $310 \mu \mathrm{m}$ (média de 229,67 $\mu \mathrm{m}$ ); comprimento variando de 320 a $590 \mu \mathrm{m}$ (média de 471,33 $\mu \mathrm{m}$ ); frequência por $\mathrm{mm}^{2}$ variando de 3 a 9 (média de 5,03). Fibras: libriformes; comprimento variando de 1.010 a $1.580 \mu \mathrm{m}$ (média de 1.281,33 $\mu \mathrm{m}$ ); largura do lume variando de 0,56 a 4,20 $\mu \mathrm{m}$ (média de 

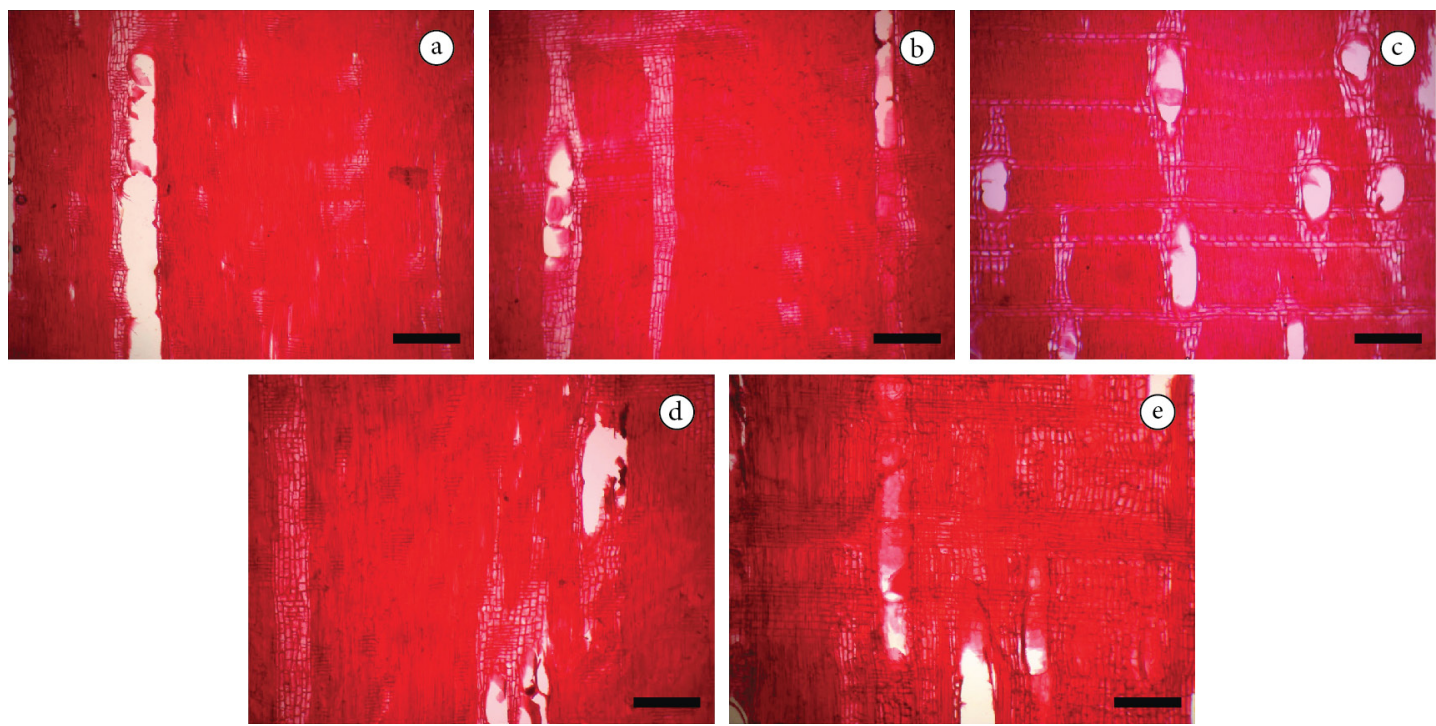

Figura 4. Vista microscópica da secção longitudinal radial. a) Bowdichia nitida Spruce ex Benth. b) Diplotropis brasiliensis (Tul.) Benth. c) Diplotropis martiusii Benth. d) Diplotropis purpurea (Rich.) Amshoff. e) Diplotropis racemosa (Hoehne) Amshoff. Barra $=500 \mu \mathrm{m}$.

Figure 4. Longitudinal radial microscopic view. a) Bowdichia nitida Spruce ex Benth. b) Diplotropis brasiliensis (Tul.) Benth. c) Diplotropis martiusii Benth. d) Diplotropis purpurea (Rich.) Amshoff. e) Diplotropis racemosa (Hoehne) Amshoff. Scale bars $=500 \mu \mathrm{m}$.

$1,36 \mu \mathrm{m})$; espessura da parede variando entre 2,52 e $8,40 \mu \mathrm{m}$ (média de 5,94 $\mu \mathrm{m}$ ). Parênquima axial: predominante paratraqueal aliforme losangular, ocorre também escasso e unilateral; cinco a oito células por strand de parênquima. Raios: corpo das células procumbentes com uma fila de células marginais quadradas e/ou eretas (Figura 4b); raios não estratificados (Figura 3b); altura em micrômetros variando de 120 a $500 \mu \mathrm{m}$ (média de 323,67 $\mu \mathrm{m}$ ); altura em número de células variando de 3 a 15 (média de 11,93); largura em micrômetros variando de 10 a $50 \mu \mathrm{m}$ (média de 32,33 $\mu \mathrm{m}$ ); largura em número de células variando de 1 a 3 (média de 2,27); frequência por mm variando de 7 a 13 (média de 8,90). Camadas de crescimento: indistintas.

\section{Diplotropis martiusii Benth.}

- Análise macroscópica

Parênquima axial: visível a olho nu; predominantemente paratraqueal aliforme losangular, formando curtas confluências, ocorre também unilateral (Figura 1c). Raios: visíveis a olho nu, não estratificados. Poros: visíveis a olho nu, porosidade difusa, arranjo com padrão indefinido, agrupados em múltiplos de 2 a mais, poros desobstruídos (Figura 1c). Camadas de crescimento: indistintas.

- Análise microscópica

Vasos: porosidade difusa; arranjo com padrão indefinido; solitários na maioria, ocorrem também múltiplos de 2 a mais; forma da secção arredondada (Figura 2c); placa de perfuração simples; pontoações intervasculares alternas; pontoações radiovasculares semelhantes às intervasculares; largura variando de 210 a $330 \mu \mathrm{m}$ (média de $284 \mu \mathrm{m}$ ); comprimento variando de 450 a $990 \mu \mathrm{m}$ (média de 696,67 $\mu \mathrm{m}$ ); frequência por $\mathrm{mm}^{2}$ variando de 2 a 6 (média de 4,40). Fibras: libriformes; comprimento variando de 1.410 a $2.370 \mu \mathrm{m}$ (média de 1.948,67 $\mu \mathrm{m}$ ); largura do lume variando de 1,12 a 10,92 $\mu \mathrm{m}$ (média de $4,87 \mu \mathrm{m}$ ); espessura da parede variando de 4,20 a 10,92 $\mu \mathrm{m}$ (média de 7,40 $\mu \mathrm{m}$ ). Parênquima axial: paratraqueal aliforme losangular, formando curtas confluências, ocorre também unilateral; três a quatro células por strand de parênquima (Figura 2c). Raios: corpo das células procumbentes com uma fila de células marginais quadradas (Figura 4c); raios não estratificados (Figura 3c); altura em micrômetros variando de 280 a $590 \mu \mathrm{m}$ (média de $418 \mu \mathrm{m}$ ); altura em número de células variando de 4 a 13 (média 
de 9,40); largura em micrômetros variando de 30 a $60 \mu \mathrm{m}$ (média de 43,67 $\mu \mathrm{m}$ ); largura em número de células variando de 1 a 3 (média de 2,03); frequência por mm variando de 5 a 11 (média de 6,73). Camadas de crescimento: indistintas.

\section{Diplotropis purpurea (Rich.) Amshoff}

- Análise macroscópica

Parênquima axial: visível a olho nu; predominantemente paratraqueal aliforme de aletas grossas, formando curtas confluências, ocorre também unilateral (Figura 1d). Raios: visíveis a olho nu, não estratificados. Poros: visíveis a olho nu; porosidade difusa; arranjo com padrão indefinido; agrupados em múltiplos de 2 a mais; em sua maioria, obstruídos por substância de cor esbranquiçada (Figura 1d). Camadas de crescimento: indistintas.

- Análise microscópica

Vasos: porosidade difusa; arranjo com padrão indefinido; solitários na maioria, ocorrem também múltiplos de 2 a mais; forma da secção arredondada (Figura 2d); placa de perfuração simples; pontoações intervasculares alternas; pontoações radiovasculares semelhantes às intervasculares; largura variando de 110 a $310 \mu \mathrm{m}$ (média de $219 \mu \mathrm{m}$ ); comprimento variando de 270 a $790 \mu \mathrm{m}$ (média de $480 \mu \mathrm{m}$ ); frequência por $\mathrm{mm}^{2}$ variando de 3 a 5 (média de $3,83)$. Fibras: libriformes; comprimento variando de 990 a $1.540 \mu \mathrm{m}$ (média de 1.279,67 $\mu \mathrm{m}$ ); largura do lume variando de 0,84 a 1,40 $\mu \mathrm{m}$ (média de 1,08 $\mu \mathrm{m}$ ); espessura da parede variando de 4,76 a 9,52 $\mu \mathrm{m}$ (média de 7,30 $\mu \mathrm{m}$ ). Parênquima axial: paratraqueal aliforme de forma losangular, formando curtas confluências; ocorre também unilateral; cinco a oito células por strand de parênquima. Raios: corpo das células procumbentes com uma fila de células marginais quadradas (Figura $4 \mathrm{~d}$ ); raios não estratificados (Figura 3d); altura em micrômetros variando de 250 a $440 \mu \mathrm{m}$ (média de $361,33 \mu \mathrm{m}$ ); altura em número de células variando de 8 a 15 (média de 12); largura em micrômetros variando de 20 a $50 \mu \mathrm{m}$ (média de $35,33 \mu \mathrm{m}$ ); largura em número de células variando de 1 a 2 (média de 1,87 ); frequência por $\mathrm{mm}$ variando de 6 a 9 (média de 7,40). Camadas de crescimento: indistintas.

\section{Diplotropis racemosa (Hoehne) Amshoff}

- Análise macroscópica

Parênquima axial: visível a olho nu; predominantemente paratraqueal aliforme confluente, ocorre também unilateral (Figura 1e). Raios: visíveis a olho nu, não estratificados. Poros: visíveis a olho nu, porosidade difusa, arranjo radial, agrupados em múltiplos de 2 a mais; apresenta alguns poros obstruídos por substância de cor escura e, em menor quantidade, por substância esbranquiçada (Figura 1e). Camadas de crescimento: indistintas.

\section{- Análise microscópica}

Vasos: porosidade difusa; em arranjo radial típico; predominantemente múltiplos de 2 a mais, ocorrem poucos solitários; forma da secção arredondada (Figura 2e); placa de perfuração simples; pontoações intervasculares alternas; pontoações radiovasculares semelhantes às intervasculares; largura variando de 90 a $360 \mu \mathrm{m}$ (média de 230,33 $\mu \mathrm{m}$ ); comprimento variando de 360 a $830 \mu \mathrm{m}$ (média de $674 \mu \mathrm{m}$ ); frequência por $\mathrm{mm}^{2}$ variando de 3 a 6 (média de $4,20)$. Fibras: libriforme; comprimento variando de 1.490 a $2.450 \mu \mathrm{m}$ (média de $1.984 \mu \mathrm{m}$ ); largura do lume variando de 0,84 a 3,92 $\mu \mathrm{m}$ (média de 1,79 $\mu \mathrm{m}$ ); espessura da parede variando de 5,88 a 12,04 $\mu \mathrm{m}$ (média de 9,45 $\mu \mathrm{m}$ ). Parênquima axial: paratraqueal aliforme confluente de aleta grossa, ocorre também unilateral; três a quatro células por strand de parênquima. Raios: corpo das células procumbentes com uma fila de células marginais quadradas e/ ou eretas (Figura 4e); raios não estratificados (Figura 3e); altura em micrômetros variando de 350 a $870 \mu \mathrm{m}$ (média de 559,33 $\mu \mathrm{m}$ ); altura em número de células variando de 11 a 31 (média de 17,87); largura em micrômetros variando de 30 a $80 \mu \mathrm{m}$ (média de $49 \mu \mathrm{m}$ ); largura em número de células variando de 2 a 4 (média de 2,77); frequência por mm variando de 4 a 8 (média de 5,53).

\subsection{Análise comparativa}

A descrição anatômica qualitativa demonstrou haver grande similaridade entre as espécies analisadas, principalmente pela presença de caracteres anatômicos, tais como presença de elementos vasculares com placa de perfuração simples, pontoações intervasculares alternas, fibras libriformes e presença de parênquima paratraqueal. Esses parâmetros foram comuns às cinco espécies analisadas e são considerados por Baas et al. (2000) como características anatômicas comuns do lenho da família Fabaceae. Metcalfe \& Chalk (1957) reiteram, 
ainda, que a presença de parênquima aliforme, por vezes confluente, é comum aos gêneros Bowdichia e Diplotropis.

Além das características anteriormente citadas como sendo comuns no lenho de Fabaceaes, observou-se também presença de parênquima axial unilateral, com células tipo strand e raios hetercelulares, tanto para as espécies do gênero Diplotropis quanto para as do gênero Bowdichia, fato que ressalta a semelhança existente entre as espécies analisadas.

No que tange aos caracteres anatômicos qualitativos avaliados, somente alguns podem ser considerados importantes na diferenciação do lenho das espécies. A espécie B. nitida pode ser distinguida das demais espécies por apresentar camadas de crescimento bem distintas, delimitadas pelo parênquima marginal com até três células de espessura, e também por apresentar raios regularmente estratificados, enquanto que as outras espécies apresentam camadas de crescimento indistintas e raios não estratificados. A madeira de $D$. racemosa pode ser diferenciada em virtude do arranjo radial característico dos vasos, bem como por apresentar raios com uma fila de células marginais quadradas e/ou eretas, enquanto que as demais espécies possuem vasos sem arranjo definido e raios com uma fileira de células marginais exclusivamente quadradas.
Quanto à análise comparativa dos parâmetros anatômicas quantitativos, observaram-se diferenças significativas $(\alpha<0,05)$ para todas as variáveis anatômicas analisadas. Entretanto, a partir da análise da Tabela 2, pode-se observar que alguns parâmetros são mais importantes na diferenciação anatômica das espécies, em virtude da maior diferença entre os valores, comparativamente: a espécie $D$. brasiliensis apresentou maior frequência de vasos por $\mathrm{mm}^{2} \mathrm{e}$ maior frequência de raios por mm linear; $B$. nitida apresentou menor comprimento dos elementos de vaso e maior largura dos raios em número de células; D. racemosa apresentou maior comprimento dos raios em micrômetros e em número de células, maior largura dos raios em micrômetros, fibras de maior comprimento, com paredes mais espessas e com maior largura; $D$. martiusii apresentou fibras com maior diâmetro do lume e $D$. purpurea apresentou a maior fração parede de fibra.

A análise de agrupamento ordenou as espécies em dois grupos distintos (Figura 5), demonstrando que, embora as cinco espécies analisadas pertençam à mesma família, sendo quatro delas do mesmo gênero, ainda assim os caracteres anatômicos quantitativos podem ser utilizados para segregar as espécies. O dendrograma (Figura 5) permite verificar, ainda, que o primeiro grupo formado reflete a maior similitude entre os parâmetros anatômicos mensurados de $D$. purpurea e $D$. brasiliensis, os quais se mantiveram

Tabela 2. Comparação das médias dos caracteres anatômicos quantitativos entre as espécies após análise estatística efetuada para: frequência dos vasos (FV), comprimento dos elementos de vasos (CV), diâmetro dos vasos (DV), frequência dos raios (FR), altura dos raios em micrômetros (AR), altura dos raios em número de células (ARc), largura dos raios em micrômetros (LR), largura dos raios e número de células (LRc), comprimento das fibras (CF), diâmetro do lume das fibras (DLF), espessura da parede das fibras (EPF), largura da fibra (LF) e fração parede da fibra (FP).

Table 2. Means comparison of the quantitative anatomical characters between species after statistical analysis performed to frequency of the vessel (FV), length of the vessel elements (CV), diameter of the vessel elements (DV), ray frequency (FR), length of rays in micrometers (AR), length of rays in number of the cells (ARc), width of ray in micrometers (LR), width of ray in number of the cells (LRc), fiber length (CF), lumen diameter of the fibers (DLF) and fiber wall thickne (EPF), fiber width (LF), fraction of the fiber wall (FP).

\begin{tabular}{|c|c|c|c|c|c|c|c|c|c|c|c|c|c|}
\hline \multirow[b]{2}{*}{ Espécie } & \multicolumn{3}{|c|}{ Vasos } & \multicolumn{5}{|c|}{ Raios } & \multicolumn{3}{|c|}{ Fibras } & \multirow[b]{2}{*}{$\begin{array}{c}\mathrm{LF} \\
(\mu \mathrm{m})\end{array}$} & \multirow[b]{2}{*}{$\begin{array}{l}\text { FP } \\
(\%)\end{array}$} \\
\hline & $\begin{array}{c}\text { FV } \\
\left(\mathbf{n}^{\mathbf{0}} \mathbf{m m}^{2}\right)\end{array}$ & $\begin{array}{c}\text { CV } \\
(\mu \mathrm{m})\end{array}$ & $\begin{array}{c}\text { DV } \\
(\mu \mathrm{m})\end{array}$ & $\begin{array}{c}\text { FR } \\
\left(\mathbf{n}^{\mathbf{0}} \mathrm{mm}\right)\end{array}$ & $\begin{array}{c}\text { AR } \\
(\mu \mathrm{m})\end{array}$ & $\begin{array}{c}\text { ARc } \\
\left(n^{\circ} \mathrm{cel}\right)\end{array}$ & $\begin{array}{c}\text { LR } \\
(\mu \mathrm{m})\end{array}$ & $\begin{array}{c}\text { LRc } \\
\left(\mathrm{n}^{\circ} \mathrm{cel}\right)\end{array}$ & $\begin{array}{c}\text { CF } \\
(\mu \mathrm{m})\end{array}$ & $\begin{array}{l}\text { DLF } \\
(\mu \mathrm{m})\end{array}$ & $\begin{array}{c}\text { EPF } \\
(\mu \mathrm{m})\end{array}$ & & \\
\hline D. martiusii & $4,4^{\mathrm{b}}$ & $696,7^{c}$ & $284^{\mathrm{b}}$ & $6,7^{b}$ & $418^{\mathrm{b}}$ & $9,4^{\mathrm{a}}$ & $43,6^{b}$ & $2,03^{\mathrm{a}}$ & $1948,6^{c}$ & $4,8^{\mathrm{b}}$ & $7,4^{\mathrm{b}}$ & $19.7^{\mathrm{c}}$ & $76^{\mathrm{a}}$ \\
\hline D. racemosa & $4,2^{\mathrm{b}}$ & $674^{c}$ & $230,3^{\mathrm{a}}$ & $5,5^{\mathrm{a}}$ & $559,3^{c}$ & $17,8^{c}$ & $49^{b}$ & $2,7^{\mathrm{b}}$ & $1984^{c}$ & $1,8^{\mathrm{a}}$ & $9,4^{c}$ & $20.7^{c}$ & $91^{\mathrm{b}}$ \\
\hline D. purpurea & $3,8^{\mathrm{a}}$ & $480^{\mathrm{b}}$ & $219^{a}$ & $7,4^{c}$ & $361,3^{\mathrm{a}}$ & $12^{\mathrm{b}}$ & $35,3^{\mathrm{a}}$ & $1,8^{\mathrm{a}}$ & $1279,6^{\mathrm{a}}$ & $1,08^{a}$ & $7,3^{\mathrm{b}}$ & $15.7^{\mathrm{b}}$ & $93^{b}$ \\
\hline D. brasiliensis & $5^{c}$ & $471,3^{\mathrm{b}}$ & $229,6^{a}$ & $8,9^{\mathrm{d}}$ & $323,6^{a}$ & $11,9^{\mathrm{b}}$ & $32,3^{\mathrm{a}}$ & $2,2^{\mathrm{a}}$ & $1281,3^{\mathrm{a}}$ & $1,79^{\mathrm{a}}$ & $5,9^{\mathrm{a}}$ & $13.2^{\mathrm{a}}$ & $87^{b}$ \\
\hline B. nitida & $3,8^{\mathrm{a}}$ & $386^{\mathrm{a}}$ & $247,3^{\mathrm{a}}$ & $7,7^{\mathrm{c}}$ & $323,3^{\mathrm{a}}$ & $13,6^{\mathrm{b}}$ & $46,3^{\mathrm{b}}$ & $3,2^{c}$ & $1713,6^{\mathrm{b}}$ & $1,64^{\mathrm{a}}$ & $7,5^{\mathrm{b}}$ & $16.7^{\mathrm{b}}$ & $90^{\mathrm{b}}$ \\
\hline
\end{tabular}

${ }_{\mathrm{a}, \mathrm{b}, \mathrm{c}}$ Médias seguidas pela mesma letra na coluna não diferem entre si pelo teste de Scott-Knott a 5\% de probabilidade. 


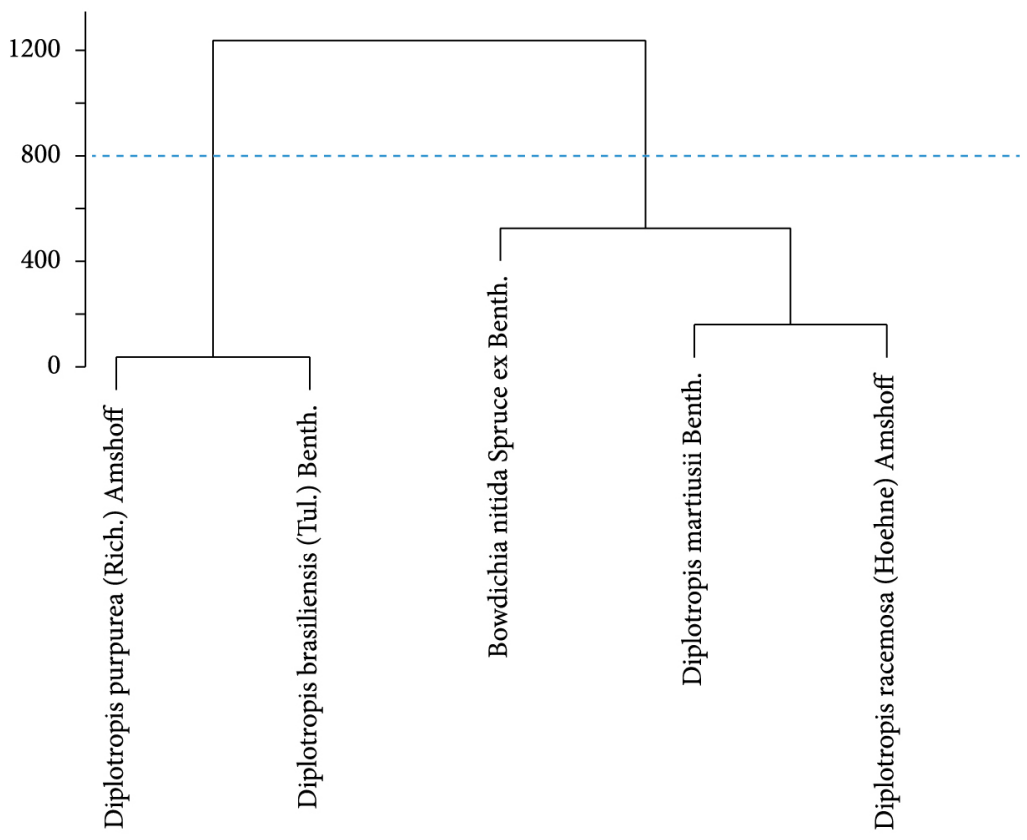

Figura 5. Análise de agrupamento. Dendrograma de similaridade entre as espécies com base nos parâmetros anatômicos quantitativos.

Figure 5. Cluster analysis. Dendrogram of similarity among species based on quantitative anatomical parameters.

mais afastados de D. racemosa e D. martiusii, que, juntamente com B. nitida, compõem o segundo grupo formado. Neste, observa-se que, apesar de a espécie $B$. nitida pertencer a um gênero botânico diferente das demais espécies analisadas, ainda assim, em virtude da semelhança de muitos de seus caracteres anatômicos, foi agrupada juntamente com D. racemosa e D. martiusii.

A análise de componentes principais (PCA) evidenciou que as características anatômicas variam dentro de fatores que, juntos, explicam 93,8\% da variância total (Figura 6). Observou-se a formação de dois grupos distintos: o primeiro formado por D. racemosa, D. martiusii e B. nitida, e o segundo formado por $D$. purpurea e $D$. brasiliensis. O primeiro grupo destaca-se principalmente pela relação positiva com o diâmetro dos elementos de vasos, a largura dos raios em micrômetros, o comprimento de fibras, a espessura da parede e a largura das fibras. Em oposição, o segundo grupo destaca-se pela relação negativa com as mesmas variáveis.

Em comparação com literatura já prescrita para as referidas espécies, a caracterização da madeira de B. nitida condiz com os trabalhos de Loureiro et al.
(2000), Mainieri (1983) e Mainieri \& Chimelo (1989). A estrutura anatômica descrita para as espécies $D$. martiusii, $D$. racemosa e $D$. purpurea assemelharam-se aos resultados observados por Mainieri (1983), em estudos com as mesmas espécies. Vasconcellos et al. (2001) também relataram características anatômicas para $D$. purpurea semelhantes às observadas neste trabalho. Para $D$. brasiliensis, não foram encontradas na literatura informações disponíveis sobre estrutura anatômica da espécie.

Do ponto de vista da utilização tecnológica da madeira, devem-se ressaltar particularmente os resultados encontrados para a espessura da parede das fibras, principalmente em virtude da influência desse parâmetro nas propriedades da madeira, notadamente aquelas relacionadas à sua resistência mecânica, tais como a densidade do lenho. Walker et al. (1993) explicam que diversas propriedades da madeira decorrem da estrutura anatômica e da orientação das microfibrilas de celulose, principalmente na parede das fibras. Nesse contexto, destaca-se o baixo valor da espessura da parede das fibras, de $5,9 \mu \mathrm{m}$, e o alto, de $9,4 \mu \mathrm{m}$, 


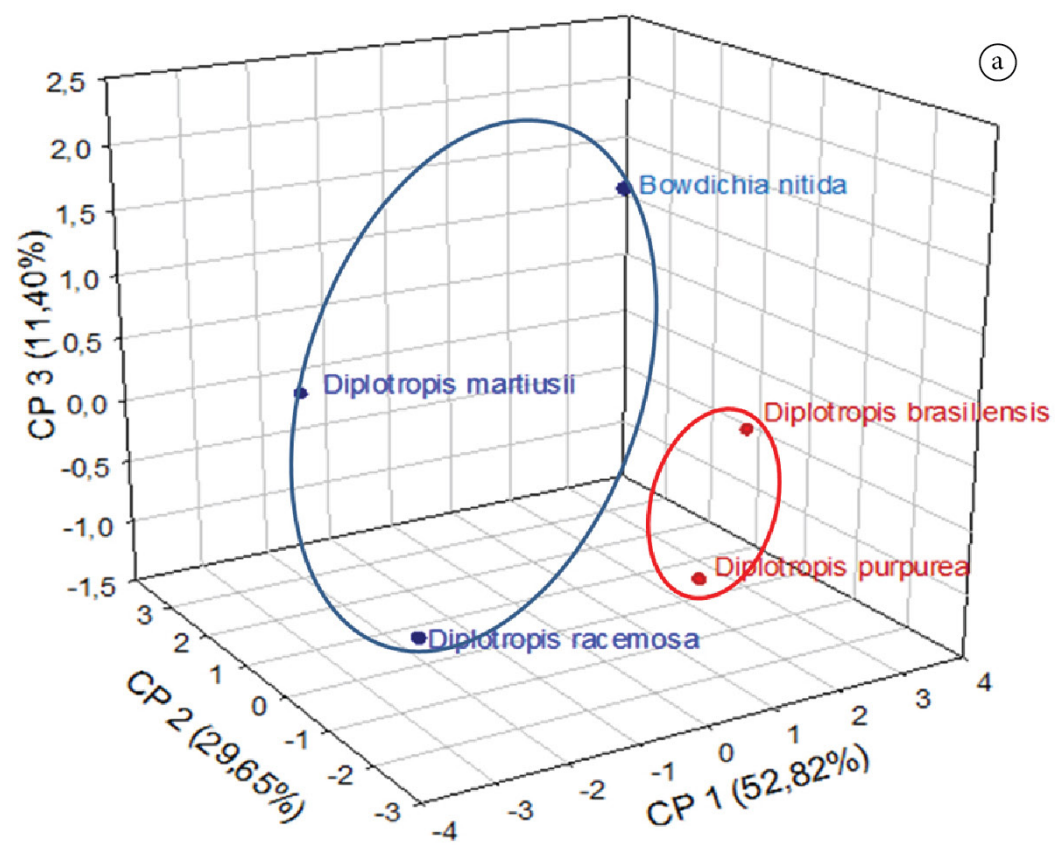

(b)

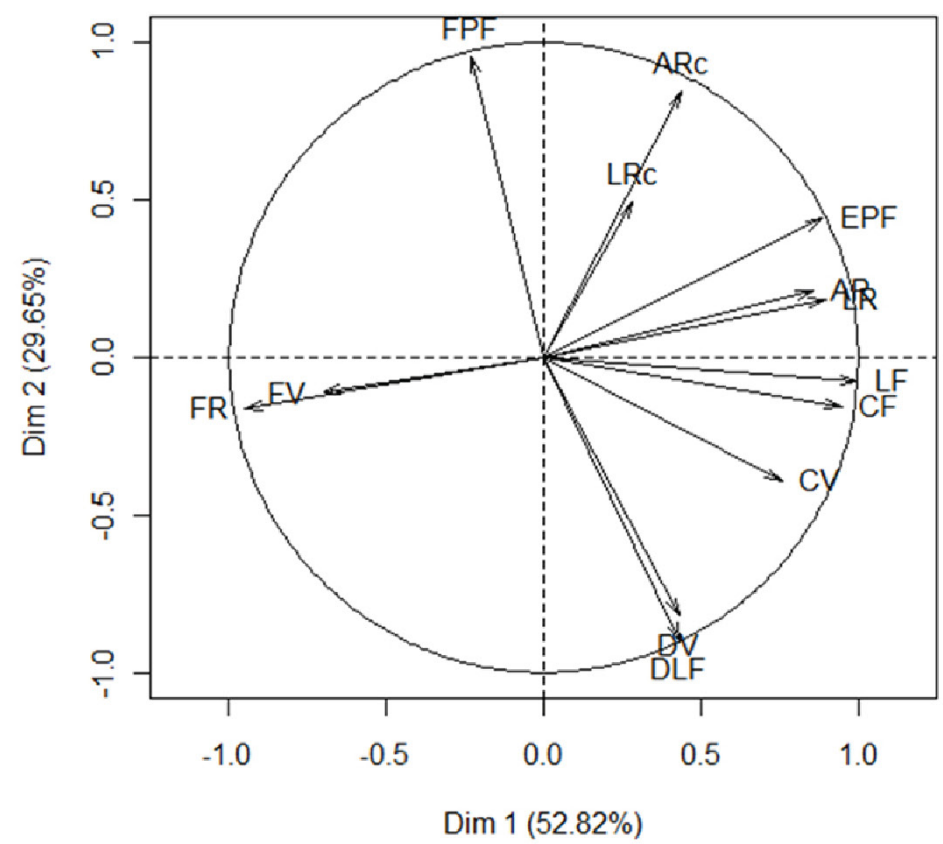

Figura 6 A-B. Análise dos componentes principais: (A) ordenação das espécies. (B) ordenação das variáveis. Frequência dos vasos (FV), comprimento dos elementos de vasos (CV), diâmetro dos vasos (DV), frequência dos raios (FR), altura dos raios em micrômetros (AR), altura dos raios em número de células (ARc), largura dos raios em micrômetros (LR), largura dos raios e número de células (LRc), comprimento das fibras (CF), diâmetro do lume das fibras (DLF), espessura da parede das fibras (EPF), largura da fibra (LF) e fração parede da fibra (FP).

Figure 6 A-B. Principal components analysis: (A) species ordination. (B) ordering of the variables. Frequency of the vessel $(\mathrm{FV})$, length of the vessel elements $(\mathrm{CV})$, diameter of the vessel elements (DV), ray frequency (FR), length of rays in micrometers (AR), length of rays in number of the cells (ARc), width of ray in micrometers (LR), width of ray in number of the cells (LRc), fiber length (CF), lumen diameter of the fibers (DLF) and fiber wall thickne (EPF), fiber width (LF), fraction of the fiber wall (FP). 
observados em $D$. brasiliensis e $D$. racemosa, respectivamente, tendo em vista a grande diferença entre os mesmos. Levando-se em consideração que todas as espécies analisadas são comercializadas em grupo, sob o mesmo nome comercial - 'sucupira' -, este fato pode-se apresentar como uma problemática, que pode vir a interferir na trabalhabilidade da madeira com diferentes ferramentas de corte e, ainda, ocasionar problemas no emprego da madeira dessas espécies para determinada finalidade.

Para Longui et al. (2009), correlações entre a estrutura anatômica e as propriedades da madeira são de extrema importância para o conhecimento e a indicação de uma ou outra espécie para determinados fins. Segundo Brasil \& Ferreira (1972), os índices primordiais na análise da qualidade da madeira são a densidade, o comprimento e a espessura da parede dos elementos fibrosos; os autores ressaltam ainda que a estrutura anatômica da madeira depende das características de crescimento da árvore e de sua carga genética, sendo qualquer modificação estrutural de grande importância na utilização industrial dessa madeira, e tendo, por isso, substancial reflexo em sua qualidade.

\section{CONCLUSÕES}

De forma geral, a descrição anatômica qualitativa demonstrou haver grande semelhança entre as cinco espécies analisadas; mostra-se possível, entretanto, diferenciar o lenho das espécies B. nitida e D. racemosa do lenho das demais.

Quanto à anatomia quantitativa do lenho das espécies, constatou-se diferença mais acentuada entre os parâmetros anatômicos mensurados, podendo-se ordenar as espécies em dois grupos distintos.

Recomenda-se maior cautela na comercialização das espécies $D$. brasiliensis e $D$. racemosa sob o mesmo nome comercial - 'sucupira' -, dada a grande diferença observada na dimensão de suas fibras.

\section{AGRADECIMENTOS}

Os autores agradecem à Xiloteca do Museu Paraense Emílio Goeldi e à Universidade do Estado do Pará, pelo apoio ao desenvolvimento do trabalho.

\section{STATUS DA SUBMISSÃO}

Recebido: 31/03/2012

Aceito: 10/10/2013

Publicado: 31/02/2014

\section{AUTOR(ES) PARA CORRESPONDÊNCIA}

\section{Luiz Eduardo de Lima Melo}

Programa de Pós-graduação em Ciência e Tecnologia da Madeira, Departamento de Ciências Florestais, Universidade Federal de Lavras - UFLA, CP 3037, Câmpus Universitário, CEP 37200-000, Lavras, MG, Brasil e-mail: luizeduardo_47@yahoo.com.br

\section{APOIO FINANCEIRO}

Conselho Nacional de Desenvolvimento Científico e Tecnológico - CNPq.

\section{REFERENNCIAS}

Baas P, Wheller E, Chase MW. Dicotyledonous wood anatomy and the APG system of angiosperm classification. Botanical Journal of the Linnean Society 2000; 134: 3-17.

Brasil MAM, Ferreira M. Variação da densidade básica e das características das fibras em Eucalyptus grandis Hill ex Maiden ao nível DAP. Instituto de Pesquisa e Estudo Florestal 1972; (4): 81-90.

Burger LM, Richter HG. Anatomia da madeira. São Paulo: Nobel; 1991. 154 p.

Franklin GL. Preparation of thin sections of synthetic resins and wood - resin composites, and a new macerating method for wood. Nature 1945; 155(3924). http://dx.doi.org/10.1038/155051a0

Gomes JI, Ferreira GC, Urbinati C. Anatomia $e$ identificação de madeiras amazônicas. Belém: Embrapa Amazônia Oriental; 2002.

Heywood VH. Flowering Plants of the World, B. T. London: Batsford LTD; 1996. 149 p.

International Association of Wood Anatomists - IAWA COMMITTEE. List of microscope features for hardwood identification. IAWA Bulletin New Series 1989; (10): 226-332.

Instituto Brasileiro do Meio Ambiente e dos Recursos Renováveis - IBAMA. Normas para procedimento em estudos de anatomia de madeira: I. angiospermae, II. gimnospermae. Brasilia; 1992. 19 p. Série Técnica n. 15. 
Jankowsky IP, Chimelo JP, Cavalcante AA. Madeiras brasileiras. Caxias do Sul-RS. Spectrum Comunicação Ltda; 1990. $171 \mathrm{p}$.

Johansen DA. Plant microtechnique. New York: McGraw-Hill; 1940. 523 p.

Kanashiro M. As Diferenças entre as Árvores. Pesquisa FAPESP 2002; (82). 33 p.

Longui EL, Lima IL, Florsheim SMB, Bufolo A. Variação anatômica radial do lenho de açoita-cavalo (Luehea divaricata) e sua influência na densidade aparente. Revista do Instituto Florestal 2009; 21(2): 181-190.

Loureiro AA, Silva MF, Alencar JC. Essências madeireiras da Amazônia. Manaus: MCT/INPA-CPPF; 2000. v. 4, 191 p.

Ludwig JA, Reynolds JF. Statistical Ecology. A primer on methods and computing. New York: John Wiley \& Sons; $1988.368 \mathrm{p}$.

Mady FTM. Técnicas para Microscopia da Madeira. Manaus: Editora da Universidade Federal do Amazonas (EDUA); 2007. 80 p.

Mainieri C. Manual de Identificação das Principais Madeiras Comerciais Brasileiras. São Paulo: Instituto de Pesquisas Tecnológicas (IPT); 1983. 241 p.

Mainieri C, Chimelo JP. Fichas das características das principais madeiras brasileiras. 2. ed. São Paulo. Instituto de Pesquisas Tecnológicas (IPT); 1989. 420 p.
Martins-da-Silva RCV. Coleta e Identificação de Espécimes Botânicos. Belém: Embrapa Amazônia Oriental; 2002. 40 p. (Série Documentos, 143).

Metcalfe CR, Chalk L. Anatomy Of The Dicotyledons: Leaves, Stem, And Wood In Relation To Taxonomy With Notes On Economic Uses. Oxford: Clarendon Press; 1957. v. 1, 806 p.

Silva MF, Lisboa PLB. Nomes vulgares de plantas amazônicas. Belém: INPA; 1977. 222 p.

Umaña CLA, Alencar JC. Comportamento fenológico da sucupira-preta (Diplotropis purourea (Rich.) Amsh. var. coriacea Amsh.), na reserva florestal Ducke. Acta amazonica 1993; 23(1): 199-211.

Vasconcellos FJ, Freitas JA, Lima VMOC, Monteiro LV, Pereira SJ. Madeiras Tropicais de Uso Industrial no Maranhão: características tecnológicas. Manaus: Instituto Nacional de Pesquisas da Amazônia (INPA), Universidade Federal do Maranhão (UFMA); 2001. $96 \mathrm{p}$.

Walker JCF, Butterfield BG, Harris JM, Langrish TAG, Uprichard JM. Primary wood processing: principles and practice. London: Chapman \& Hall; 1993. 595 p. http:// dx.doi.org/10.1007/978-94-015-8110-3

Zar JH. Biostatistical analysis. 3. ed. Prentice Hall; 1996. 